\title{
Orbital and physical properties of planets and their hosts: new insights on planet formation and evolution
}

\author{
V. Zh. Adibekyan ${ }^{1}$, P. Figueira ${ }^{1}$, N. C. Santos ${ }^{1,2}$, A. Mortier ${ }^{1,2}$, C. Mordasini ${ }^{3}$, \\ E. Delgado Mena ${ }^{1}$, S. G. Sousa ${ }^{1,2,4}$, A. C. M. Correia ${ }^{5,6}$, G. Israelian ${ }^{4,7}$, and M. Oshagh ${ }^{1,2}$ \\ ${ }^{1}$ Centro de Astrofísica da Universidade do Porto, Rua das Estrelas, 4150-762 Porto, Portugal \\ e-mail: Vardan. Adibekyan@astro.up.pt \\ 2 Departamento de Física e Astronomia, Faculdade de Ciências da Universidade do Porto, 4169-007 Porto, Portugal \\ 3 Max-Planck-Institut für Astronomie, Königstuhl 17, 69117 Heidelberg, Germany \\ ${ }^{4}$ Instituto de Astrofísica de Canarias, 38200 La Laguna, Tenerife, Spain \\ 5 Departamento de Física, I3N, Universidade de Aveiro, Campus de Santiago, 3810-193 Aveiro, Portugal \\ 6 ASD, IMCCE-CNRS UMR8028, Observatoire de Paris, UPMC, 77 Avenue Denfert-Rochereau, 75014 Paris, France \\ 7 Departamento de Astrofísica, Universidad de La Laguna, 38206 La Laguna, Tenerife, Spain
}

Received 27 August 2013 / Accepted 11 November 2013

\begin{abstract}
Aims. We explore the relations between physical and orbital properties of planets and properties of their host stars to identify the main observable signatures of the formation and evolution processes of planetary systems.

Methods. We used a large sample of FGK dwarf planet-hosting stars with stellar parameters derived in a homogeneous way from the SWEET-Cat database to study the relation between stellar metallicity and position of planets in the period-mass diagram. We then used all the radial-velocity-detected planets orbiting FGK stars to explore the role of planet-disk and planet-planet interaction on the evolution of orbital properties of planets with masses above $1 M_{\text {Jup }}$.

Results. Using a large sample of FGK dwarf hosts we show that planets orbiting metal-poor stars have longer periods than those in metal-rich systems. This trend is valid for masses at least from $\approx 10 M_{\oplus}$ to $\approx 4 M_{\text {Jup }}$. Earth-like planets orbiting metal-rich stars always show shorter periods (fewer than 20 days) than those orbiting metal-poor stars. However, in the short-period regime there are a similar number of planets orbiting metal-poor stars. We also found statistically significant evidence that very high mass giants (with a mass higher than $4 M_{\text {Jup }}$ ) have on average more eccentric orbits than giant planets with lower mass. Finally, we show that the eccentricity of planets with masses higher than $4 M_{\text {Jup }}$ tends to be lower for planets with shorter periods.

Conclusions. Our results suggest that the planets in the $P-M_{\mathrm{P}}$ diagram are evolving differently because of a mechanism that operates over a wide range of planetary masses. This mechanism is stronger or weaker, depending on the metallicity of the respective system. One possibility is that planets in metal-poor disks form farther out from their central star and/or they form later and do not have time to migrate as far as the planets in metal-rich systems. The trends and dependencies obtained for very high mass planetary systems suggest that planet-disk interaction is a very important and orbit-shaping mechanism for planets in the high-mass domain.
\end{abstract}

Key words. planetary systems - planet-disk interactions - planets and satellites: formation - stars: fundamental parameters

\section{Introduction}

The history of the discovery of extrasolar planets is a story of challenges for theories of planet formation. The first extrasolar planet orbiting a main sequence $\operatorname{star}^{1}, 51$ Peg b (Mayor \& Queloz 1995), proved to be very much at odds with the theory of formation of our own solar system (SS), our only reference at the time. As the number of planets increased and several close-in (hot-) Jupiters were found, it became clear that a new formation mechanism was necessary. An in situ formation of these planets was unlikely, because of the insufficient disk mass close to the star. However, their presence at an orbit of $\sim 0.05$ AU could be reconciled with a farther out formation by invoking migration during or after the formation process (e.g. Lin et al. 1996). The SS formation theories suggested that giant planets preferentially formed close to the ice-line (especially in low-metallicity disks), where water is condensed into ice and the necessary building blocks for the formation of planets could be found in large

\footnotetext{
1 The first detection of two terrestrial-mass exoplanets around a pulsar PSR B1257+12 was announced in 1992 (Wolszczan \& Frail 1992).
}

quantities (e.g. Ida \& Lin 2008). An important breakthrough was thus to explain the existence of hot-Jupiters by considering core-accretion simultaneously with disk-driven migration (e.g. Pollack et al. 1996; Alibert et al. 2005; Mordasini et al. 2009a) while reproducing several observational trends (Mordasini et al. $2009 b$ ). It explained the correlation of the presence of giant planets with stellar metallicity (e.g. Santos et al. 2001, 2004; Fischer \& Valenti 2005; Sousa et al. 2011), a correlation that could not be explained by the alternative formation model, gravitational instability (e.g. Boss 1998).

However, when the formation paradigm seemed to be complete, an unexpected result emerged. The discovery of hotJupiters whose orbital plane was misaligned with the stellar rotation axis (e.g. Hébrard et al. 2008; Triaud et al. 2010; Brown et al. 2012) cast serious doubts on disk-driven migration as the mechanism responsible for the hot-Jupiters. In particular, the occurrence of retrograde planets required an additional mechanism. Two main solutions put forward were Kozai cycles plus tidal perturbations (e.g. Wu \& Murray 2003; Fabrycky \& Tremaine 2007; Correia et al. 2011) and planet-planet scattering 
(e.g. Rasio \& Ford 1996; Beaugé \& Nesvorný 2012). The implication was that planets first form in the disk at several AU, then undergo gravitational perturbations that increase the eccentricity to very high values, and finally reduce their semi-major axis by tidal interactions with the star that simultaneously dampen the eccentricity to zero by conservation of the orbital angular momentum ${ }^{2}$.

To study the main mechanism responsible for the presence of close-in Jupiters, Socrates et al. (2012) and Dawson et al. (2012) analyzed the eccentricity distribution of proto-hot Jupiters. The paucity of super-eccentric proto-hot Jupiters observed in the Kepler sample allowed the latter authors to conclude that disk migration is the dominant mechanism that produces hot-Jupiters, although some of these planets might be perturbed to higheccentricity orbits by interactions with planetary companions.

It is interesting to note how the signatures of the formation and evolution mechanisms relate to the mass of the planet. There is an ongoing debate on whether the core-accretion model can reproduce the properties of Earth-mass planets (Fortier et al. 2013). Recent works suggested that unlike for more massive planets, these systems might have been formed in situ (e.g. Hansen \& Murray 2012; Chiang \& Laughlin 2013). While these types of studies are still in their infancy, they already reproduce some of the most common features of the low-mass planet systems, such as being dynamically packed and showing low inclination and eccentricities (Lovis et al. 2011; Figueira et al. 2012). On the other hand, planets with masses between $0.05 M_{\text {Jup }}$ and $20 M_{\text {Jup }}$ have been characterized by core-accretion and migration formation, and evolution models and can be compared quite well with the observations.

Evidence for the migration processes involved in the formation of short-period planets has previously been discussed in the literature. For instance, several works suggested discontinuities in observables inside this mass range. Beaugé \& Nesvorný (2013) presented tentative evidence that the smallest $\left(<4 R_{\oplus}\right)$ Kepler planetary candidates orbiting metal-poor stars show a period dependence (see Sect. 2); Dawson \& Murray-Clay (2013) argued that giant planets orbiting metal-rich stars show signatures of planet interaction. The first result provides compelling evidence for the importance of migration and accretion, the second for planet-planet interaction. For the upper end of the mass population, composed of planets with masses above $4 M_{\text {Jup }}$, a long discussion has been ongoing on whether this population resembles more closely lower-mass planets or shares properties with stellar binaries (e.g. Udry et al. 2002; Halbwachs et al. 2005). The eccentricity distribution of this planetary population has been shown to be similar to that of binaries, and some doubts were cast on core-accretion as their formation mechanism (Ribas \& Miralda-Escudé 2007).

In this work we explore the relations and correlations between planetary mass and orbital parameters, namely period and eccentricity, and how they relate to metallicity, for a wide range of planetary masses. We start by extending the work of Beaugé \& Nesvorný (2013) for higher planetary masses in Sect. 2, and in Sect. 3 we explore the properties of planets with masses higher than $4 M_{\text {Jup }}$. We summarize our main results and their implications on our understanding of planet formation in Sect. 4.

We note that in this work the masses of the planets $\left(M_{\mathrm{P}}\right)$ are indeed minimum masses, so that in some cases the "true"

\footnotetext{
2 However, there are several other mechanisms that can tilt a star relative to its protoplanetary disk: gravitational torques from massive distant bodies (Batygin 2012) or angular momentum transport within the host star (Rogers et al. 2012).
}

planet mass may be significantly higher. Nevertheless, statistical analyses show that the distribution of $M_{\mathrm{P}} \sin i$ values is similar to that of $M_{\mathrm{P}}$ values (e.g. Jorissen et al. 2001; but see the discussion by Lopez \& Jenkins 2012, for low-mass planets). In fact, the average factor of overestimation is only 1.27, assuming a random distribution of the inclination.

We would like to say a word of caution regarding the significance estimates quoted in the next sections. In general, if one uses the same data to test several hypotheses, the results can be affected by the multiple-testing problem ${ }^{3}$. However, since throughout our study we dealt with only a few parameters and the significance levels of the results are very high, it can be shown by simple algebra that the current proposed trends are significant, although we acknowledge the possibility that the quoted significance levels might be optimistic.

\section{Period-mass diagram and metallicity}

Recently, Beaugé \& Nesvorný (2013) analyzed the distribution of planets in the orbital period $(P)$ versus planetary radius $(R)$ diagram and $P$ versus $M_{\mathrm{P}}$ diagram. They found a lack of smallsize/low-mass planets $\left(R \lesssim 4 R_{\oplus}, M_{\mathrm{P}} \lesssim 0.05 M_{\text {Jup }}\right)$ with periods $P<5$ days around metal-poor stars. They also found a paucity of sub-Jovian size/mass planets around metal-poor stars with periods shorter than 100 days. They explained these observed trends with a delayed formation and less planetary migration in metal-poor disks. Interestingly, the authors found no significant correlation between metallicity and the position of giant planets in the $(P, R)$ or $\left(P, M_{\mathrm{P}}\right)$ diagram. In this section we extend the analysis of these authors to higher-masses using a large sample of FGK dwarf stars $\left(M_{*}>0.5 M_{\odot}\right)$ with stellar parameters derived in a homogeneous way (SWEET-Cat: a catalog of stellar parameters for stars with planets: Santos et al. 2013) ${ }^{4}$.

Figure 1 shows the $\left(P, M_{\mathrm{P}}\right)$ diagram for planets detected with different techniques that orbit stars with different evolutionary states. In the plot we separate the planets according to the metallicity of their host stars. As one can see in the bottom - left panel of the figure, most of the transiting planets have very short periods and their hosts are confined to a narrow range of metallicity (their hosts are mostly metal-rich). It is also very clear that planets around evolved stars have a very small range of $P$ and their hosts show very different metallicity distributions compared with their unevolved counterparts (for example, about $40 \%$ of planets around evolved stars have $[\mathrm{Fe} / \mathrm{H}]<$ -0.1 dex, while fewer than $20 \%$ of planets orbiting dwarf stars have similarly low metallicity). This difference in metallicity distribution is probably due to selection biases in evolved stellar samples that are used for planets searches (Mortier et al. 2013). We conclude therefore that these samples have such strong selection and detection biases that they are not suitable for our analysis. We therefore concentrate the discussion on dwarf stars with planets detected by radial velocity when we discuss the impact of the planet host's metallicity.

The right panel of Fig. 1 shows RV-detected planets orbiting FGK dwarfs on the $P-M_{\mathrm{P}}$ plane in which we separate lowand high-metallicity hosts at a threshold of $-0.1 \mathrm{dex}$, which is the average metallicity of the stars in the solar neighborhood (e.g. Adibekyan et al. 2012c). The same diagram, but for planets with masses lower than $0.1 M_{\text {Jup }}$ is shown in the top panel of

\footnotetext{
3 See

http://en.wikipedia.org/wiki/Multiple_comparisons

4 https://www .astro.up.pt/resources/sweet-cat
} 

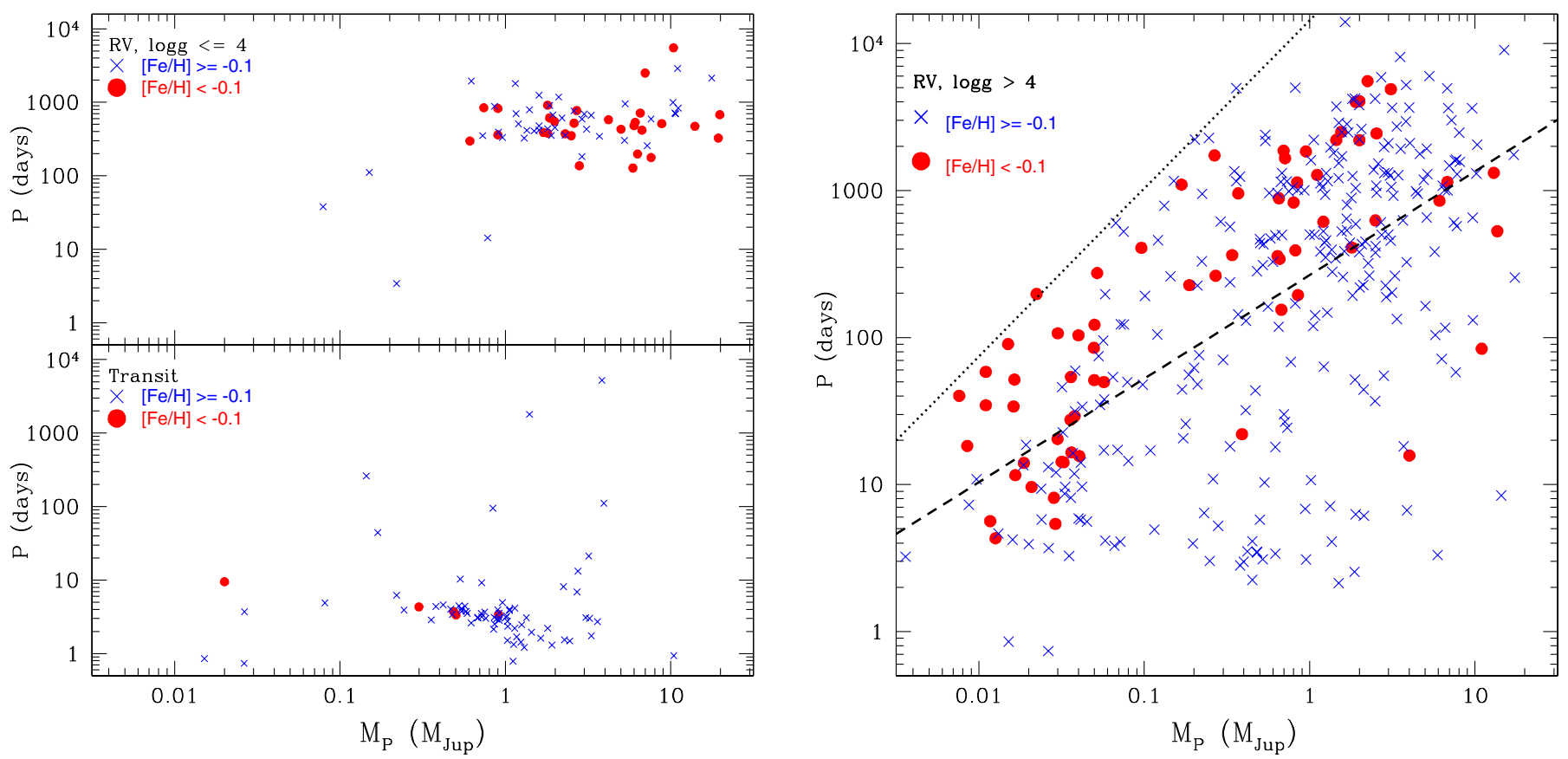

Fig. 1. Planet distribution in the $\left(P, M_{\mathrm{P}}\right)$ diagram. In the top-left panel only the planets around evolved stars with $\log g \leq 4$ dex are shown. In the bottom-left panel we plot only the planets detected with the transiting method, and in the right panel we present the planets orbiting FGK dwarfs $(\log g>4 \mathrm{dex})$. The dotted line represents the approximate (empirical) detection limit for planets, and the dashed line is the linear fit for the full sample.

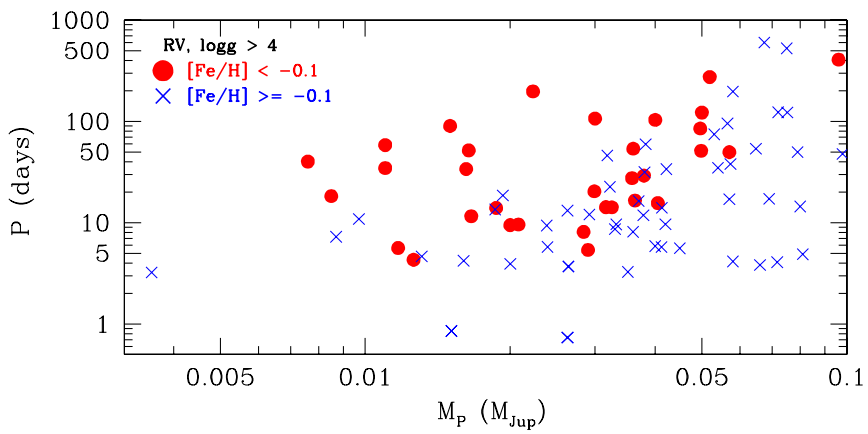

the number of planets orbiting metal-poor stars in a given interval of mass from $0.03 M_{\text {Jup }}$ to $M_{\mathrm{P}}(\max )$, and we randomly drew the same number of points from the planetary sample around metal-rich stars. Then we counted the number of planets orbiting metal-poor and metal-rich stars below the line of the linear fitting of the full data $\left(P, M_{\mathrm{P}}\right.$ of all the planets in our initial sample, i.e., metal-poor and metal-rich planets with masses from 0.0036 to $17.4 M_{\text {Jup }}$ ). We repeated the entire process $10^{5}$ times. By comparing the average number (and using the standard deviation) of metal-rich planets found below the fitted line with the number of metal-poor planets (again bellow the fitted line), we evaluated the significance (the $z$-score $-n \sigma$ ) of the statement that the

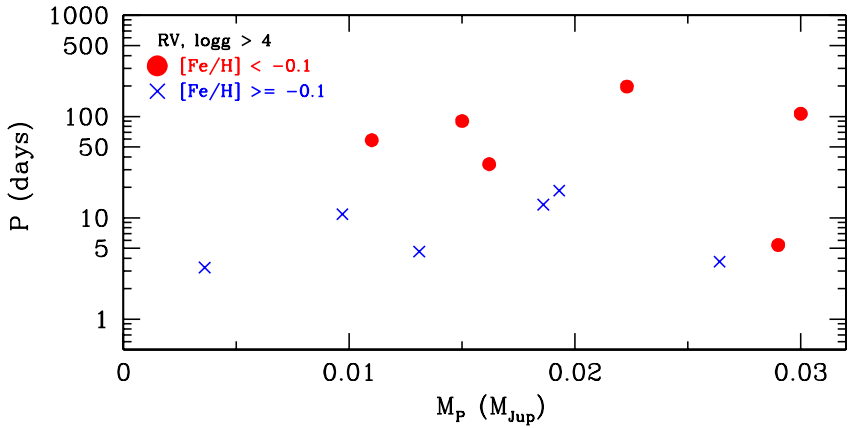
two metal-poor and metal-rich populations have different distributions of $\left(P, M_{\mathrm{P}}\right)^{5}$.

The $M_{\mathrm{P}}(\max )$ originally was $1 M_{\mathrm{Jup}}$, which we later varied up to the highest value of the planet mass in the sample. The metallicity boundary was also considered as variable $(-0.10$, -0.05 , and $0.00 \mathrm{dex})$. We found that the significance of the correlation decreases with the increase of $M_{\mathrm{P}}(\max )$ and interestingly, after $M_{\mathrm{P}}(\max )=4 M_{\text {Jup }}$, the significance decreased faster. From the right panel of Fig. 1 one can see that almost all the planets with masses higher than $4 M_{\text {Jup }}$ orbiting metal-poor stars lie below the dashed line. Depending on the combination of the parameters $\left([\mathrm{Fe} / \mathrm{H}]\right.$ and $\left.M_{\mathrm{P}}(\max ) \leq 4 M_{\text {Jup }}\right)$, the significance of our

Fig. 2. Same as Fig. 1 right panel, but only for planets with $M_{\mathrm{P}}<$ $0.1 M_{\text {Jup }}\left(\right.$ top panel). The $\left(P, M_{\mathrm{P}}\right)$ diagram for the longest-period planet in the system with $M_{\mathrm{P}}<0.03 M_{\text {Jup }}$ (bottom panel).

Fig. 2. As one can see, for a fixed planetary mass, most of the planets around metal-poor stars are constrained to longer periods. This tendency appears to be valid from about $0.03 M_{\text {Jup }}$ to about $4 M_{\text {Jup }}$ and confirms and extends the results of Beaugé \& Nesvorný (2013) to higher masses.

To evaluate the statistical significance of the observed trend we performed a simple Monte Carlo (MC) test. First, we counted results varies from $\{4.4 \sigma$ to $6.1 \sigma$, which means that it is always significant.

To ensure that the observed trend is genuinely valid for the higher-mass domain and is not just influenced by the previously reported trend for low-mass planets (Beaugé \& Nesvorný 2013), we repeated the test, selecting $M_{\mathrm{P}}(\mathrm{min})$ in the range from 0.1 to 0.3 Jupiter mass and excluding the planets with $M_{\mathrm{P}}>4 M_{\mathrm{Jup}}$. We confirm the previous result at a $1.8 \sigma$ to $3.7 \sigma$ significance

5 We note that the quoted significance levels are the result of a onesided test, since the test by construction is one-sided. 
level (depending on the combination of fixed parameters, i.e., $\left.[\mathrm{Fe} / \mathrm{H}], M_{\mathrm{P}}(\mathrm{min})\right)$ : the significance was lowest when $M_{\mathrm{P}}(\mathrm{min})$ is 0.3 Jupiter mass and the $M_{\mathrm{P}}(\max )$ is $1 M_{\text {Jup }}$ (when the number of planets in the subsample is the smallest).

Furthermore we made a similar test, but instead of counting the planets below the fitted line, we considered a horizontal line (constant period). Varying this horizontal line from about 80 to 400 days, we again found that the result is always higher than $2.1 \sigma$ (reaching up to $3.2 \sigma$ ). These tests show that the results do not depend significantly on the position of the separation (fitted line or constant period), and they suggest that the findings by Beaugé \& Nesvorný (2013) about the lack of small-size planets around metal-poor stars at short periods extend up to 4 Jupiter masses.

\subsection{Earth-like planets}

All the planets with masses bellow $0.03 M_{\text {Jup }}$ (about $10 M_{\oplus}$ ) orbiting metal-rich stars have short periods of fewer than 18 days (see top panel of Fig. 2). It is difficult to understand why these low-mass planets do not have longer periods, such as we observe for planets in the metal-poor systems. One of the reasons for this disposition can have a dynamical character. Most of the planets orbiting metal-poor stars belong to the multiple systems where almost all the planets have low masses $\left(M_{\mathrm{P}}<0.03 M_{\mathrm{Jup}}\right)$, while planets in the multiple systems orbiting metal-rich stars have higher-mass planetary companions with longer periods. However, in the bottom panel of Fig. 2, where we show the distribution of the planets with the longest period in the system, one can see that there are six planets orbiting metal-rich stars without a (detected) higher-mass longer-period companion. This means that at least for the planets presented in the plot, the shorter periods are not a result of interaction with higher-mass longerperiod companions. Another explanation of the lack of metalrich low-mass planets with long periods might be a detection limit, since metal-rich planet hosts are on average slightly more massive and hotter than their metal-poor counterparts. However, the mentioned differences are very small and probably cannot be responsible for the observed short periods.

If there is no detection bias in the sample, then the observed distributions of metal-poor and metal-rich planets in the $P-M_{\mathrm{P}}$ diagram could mean that Earth-like planets orbiting metal-rich stars preferably migrate or form close to their parent stars, while planets in the metal-poor systems form at a wider range of the semi-major axis or do not always migrate.

In the small range of periods (fewer than 18 days) where metal-rich planets orbit, there are also similar planets orbiting metal-poor stars, and their period distribution seems similar (excluding the planets with longer periods orbiting metal-poor stars), although it is difficult to evaluate the significance because of the small number of planets.

We note that the number of planets discussed in this subsection is small, therefore, the results and conclusions regarding them should be considered with caution.

\section{2. $[\mathrm{Ref} / \mathrm{H}]$ vs. $[\mathrm{Fe} / \mathrm{H}]$}

Typically, $[\mathrm{Fe} / \mathrm{H}]$ is used as a proxy of overall metallicity for stars, but if a star has peculiar chemistry or is enhanced by some individual elements compared to iron, then the $[\mathrm{Fe} / \mathrm{H}]$ index will differ from the total metallicity $[\mathrm{M} / \mathrm{H}]$. Adibekyan et al. (2012a,b) showed that most of the planet-host stars with low-iron content are enhanced by $\alpha$-elements, including $\mathrm{Mg}$ and $\mathrm{Si}$ which are fairly abundant and have condensation temperatures similar to iron (Lodders 2003; Lodders et al. 2009). Beaugé \& Nesvorný (2013) recently questioned how their result would be affected if one used the $[\mathrm{Ref} / \mathrm{H}]$ index $^{6}$ instead of $[\mathrm{Fe} / \mathrm{H}]$.

To quantify the effect of $[\mathrm{Ref} / \mathrm{H}]$ on our result we used the HARPS sample of 135 planet-host stars with detailed and precise chemical abundances derived in Adibekyan et al. (2012c). In this sample 32 planet hosts have $[\mathrm{Fe} / \mathrm{H}]$ lower than -0.1 dex. We found that in the mentioned metallicity region the $[\mathrm{Ref} / \mathrm{H}]$ is on average higher than $[\mathrm{Fe} / \mathrm{H}]$ by $\approx 0.05$ dex: about $0.00 \mathrm{dex}$ for stars with $[\mathrm{Fe} / \mathrm{H}] \approx-0.1$ dex and up to 0.15 dex for the stars with $[\mathrm{Fe} / \mathrm{H}] \approx-0.6$ dex. Only two out of the mentioned 32 planet hosts with $[\mathrm{Fe} / \mathrm{H}]<-0.1$ dex show $[\mathrm{Ref} / \mathrm{H}]>-0.1 \mathrm{dex}$, but both cases are very close to the boundary $(-0.09$ and -0.08 dex $)$. Recalling that our results do not depend on the metallicity boundary, and assuming that the HARPS planet host sample is representative for the whole sample discussed above, we can conclude that using the $[\mathrm{Ref} / \mathrm{H}]$ index instead of $[\mathrm{Fe} / \mathrm{H}]$ will not affect our results.

\subsection{Role of $[\mathrm{Fe} / \mathrm{H}]$ on the position of planets in the $P-M_{P}$ diagram. Discussion}

The most important insights from our tests and results come from the continuity or discontinuity of the extrasolar planet population as a function of key parameters. The correlation between period, mass, and metallicity presented in Fig. 1 suggests the existence of a mechanism that affects a wide range of masses, from $10 M_{\oplus}$ to $4 M_{\text {Jup }}$, and that depends on metallicity. Dawson \& Murray-Clay (2013) showed that high-eccentricity Jupitermass planets with semi-major axes $(a)$ between 0.1 and 1 AU were preferably found orbiting metal-rich stars; the authors interpreted this finding as evidence for planet-planet scattering on a population created by smooth migration. The main assumption was that $[\mathrm{Fe} / \mathrm{H}]$ will not significantly affect the $P-M_{\mathrm{P}}$ relationship as created by core-accretion+migration (see Mordasini et al. 2012, for details), and thus the observed difference was created exclusively by dynamical interactions. They also noted that beyond $1 \mathrm{AU}$, the metal-rich and metal-poor samples have a similar eccentricity distribution with the explanation that planets with $a>1$ AU may have formed where we observe them.

We applied a Kolmogorov-Smirnov (K-S) test for the samples of giant planets $\left(M_{\mathrm{P}}>0.1 M_{\text {Jup }}\right.$ and with $P>10$ days $)$ orbiting metal-poor $([\mathrm{Fe} / \mathrm{H}]<-0.1 \mathrm{dex})$ and metal-rich $([\mathrm{Fe} / \mathrm{H}] \geq$ -0.1 dex) dwarf stars and found that their eccentricity distributions are similar (K-S probability $\approx 0.63$ ). To test whether these distributions are similar for different period regimes we made two subsamples of planets with periods $10<P \leq 300$ days (300 days corresponds to $\sim 1 \mathrm{AU}$, which was the upper limit of $a$ in Dawson \& Murray-Clay 2013), and with $P>300$ days. Since the number of planets orbiting around metal-poor stars with $[\mathrm{Fe} / \mathrm{H}] \geq-0.1$ dex is small (only seven stars), we changed the metallicity boundary from -0.1 to 0.0 dex. We note that this was also the metallicity boundary chosen by Dawson \& Murray-Clay (2013). The applied K-S test predicts $P_{\mathrm{KS}}=0.10$ and $P_{\mathrm{KS}}=0.97$ that metal-poor and metal-rich populations of planets with short and long periods have the same eccentricity distribution, respectively. Thus, we confirm the results of Dawson \& Murray-Clay (2013) for "close-in" giants. We note

\footnotetext{
6 This index was proposed by Gonzalez (2009) and quantifies the mass abundances of refractory elements $(\mathrm{Mg}, \mathrm{Si}$ and $\mathrm{Fe})$ relevant for planet formation.
} 
that for the whole range of periods ( $P>10$ days) the K-S statistics delivers $P_{\mathrm{KS}} \approx 0.66$ for the similarity of eccentricity distribution of planets orbiting stars with $[\mathrm{Fe} / \mathrm{H}]<0.0$ dex and $[\mathrm{Fe} / \mathrm{H}] \geq 0.0$ dex. The test implies that the $P-M_{\mathrm{P}}$ distribution of the metal-poor population (at least for planets with periods longer than 300 days) results from formation and migration and has no dynamical character. The fact that massive planets at low$[\mathrm{Fe} / \mathrm{H}]$ are found farther out implies that these planets probably are only able to form beyond the ice-line for low-metallicities (Mordasini et al. 2010, 2012) ${ }^{7}$.

The similarity of the period distribution of short-period Earth-like planets $\left(M_{\mathrm{P}}<10 M_{\oplus}\right)$ around metal-rich and metalpoor stars probably means that low-mass planets can be formed at small semi-major axes even at low metallicities because a sufficient amount of protoplanetary mass is locally available. At the same time, the fact that planets orbiting metalrich stars are observed only at short periods might imply that these planets form close to their central stars or ordinarily migrate. However, we note that the statistical significance of the last results are not evaluated because of small number of planets.

It is also interesting to note that if we compare metal-poor stars with their metal-rich counterparts, there is a dearth of planets with mass around $0.1 M_{\text {Jup }}$ orbiting the metal-poor stars (see also Beaugé \& Nesvorný 2013). In the paradigm of Dawson \& Murray-Clay (2013), these Neptune-mass planets are preferentially formed farther out, (again probably around or beyond the ice-line), and move into smaller orbits because of planet interaction. Interestingly, this is qualitatively in line with results from microlensing surveys, which state that cold Neptunes are indeed common (Sumi et al. 2010; Gaudi 2012). However, a quantitative analysis would require an assessment and correction for detection bias, which is far beyond the scope of this paper.

\section{Orbital and physical properties of very massive planets}

In the previous section we noted that the planets with very high masses show different dependencies on metallicity in the $\left(P, M_{\mathrm{P}}\right)$ diagram from their less massive counterparts. This may be related both to formation and to post-formation processes (interaction with disk or interaction between planets). To form a very high planetary mass, a critical core must grow, a process that takes longer in a low-metallicity environment (Mordasini et al. 2012). The time scale of the orbital evolution of planets also depends on the properties of the protoplanetary disk and on other initial conditions (e.g. Xiang-Gruess \& Papaloizou 2013; Bitsch et al. 2013). To determine whether this difference between highand low-mass planets in the $\left(P, M_{\mathrm{P}},[\mathrm{Fe} / \mathrm{H}]\right)$ diagram is reflected on other parameters as well, we compared the physical and orbital parameters of planets with masses from 1 to $4 M_{\text {Jup }}$ to those with masses higher than $4 M_{\text {Jup }}{ }^{8}$. For this analysis we used all the RV-detected planets orbiting FGK stars from the Extrasolar Planets Encyclopaedia (Schneider et al. 2011) ${ }^{9}$.

\footnotetext{
7 This would in turn imply that migration does not significantly change the semi-major axis of low-metallicity planets. This last point seems to be in line with what is expected from the models, but depends on the definition of the ice-line, among other aspects.

8 We chose to establish a low-mass cut-off because one can assume that at least the mechanism of formation of the planets in this two planetary mass regimes is similar.

9 http://www. exoplanet.eu
}

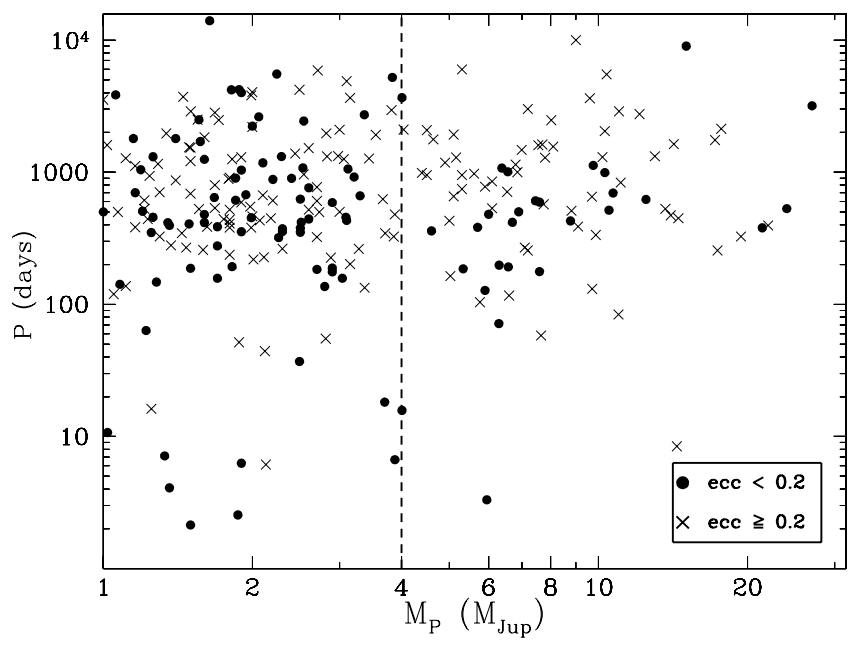

Fig. 3. $\left(P, M_{\mathrm{P}}\right)$ diagram for the all RV detected planets with $M_{\mathrm{P}}>1 M_{\text {Jup }}$ orbiting FGK stars from exoplanet.eu. Planets with high- and loweccentricity orbits are presented by crosses and circles, respectively.

\subsection{Orbital eccentricity}

The first significant correlation we found is that very massive planets are more eccentric than their lower-mass counterparts. This correlation is illustrated in Fig. 3. Sixty-one out of 90 planets $(66.3 \pm 5.0 \%)$ with $M_{\mathrm{P}}>4 M_{\text {Jup }}$ have $e>0.2$, while only 94 out of $173(54.3 \pm 3.8 \%)$ planets with masses between 1 and $4 M_{\text {Jup }}$ are similarly eccentric. To assess the statistical significance of the difference in observed eccentricity distributions between low- and high mass planets we performed a K-S test. The K-S statistics predict a $0.0026(\approx 3 \sigma)$ probability $\left(P_{\mathrm{KS}}\right)$ that the two subsamples come from the same underlying distribution of $e$. We note that when we consider only the planets orbiting FGK dwarf stars the difference remains significant; $80.4 \pm 5.8$ and $62.1 \pm 4.6$ percent of eccentric planets with masses higher than $4 M_{\text {Jup }}$ and with $1 M_{\text {Jup }}<M_{\mathrm{P}}<4 M_{\text {Jup }}$, respectively. To test whether our result is affected by close-in planets, which mostly have circular orbits due to tide effects (see e.g. Ford \& Rasio 2006), we established a minimum period cut-off at 10, 50, and 100 days (although from Fig. 3 it is clear that most of the RV-detected planets have $P>100$ days). The K-S probabilities for the three cases were $0.004,0.0036$, and 0.0022 respectively, which allows us to conclude firmly that this is not the case.

Udry et al. (2002) and Ribas \& Miralda-Escudé (2007) had already found a marginal tendency for low-mass planets $\left(M_{\mathrm{P}}<\right.$ $4 M_{\text {Jup }}$ ) to be less eccentric than more massive planets and binary stars. We note that in these two studies the authors did not separate very small planets (Earth-like or Neptune-like) from the massive gaseous planets ${ }^{10}$. However, Udry et al. (2002) noted that when one restricts the sample to periods longer than 50 days (avoiding the circularization through tidal interactions), the difference in eccentricities between low- and high-mass planets disappears.

It has been suggested that the eccentricity of planets can be increased through the planet-disk interaction under favorable conditions and especially if the planetary mass is very high (Papaloizou et al. 2001; Kley \& Dirksen 2006; D'Angelo et al. 2006). Very recently, Bitsch et al. (2013), performing isothermal 3D simulations, showed that the eccentricity of planets in

${ }^{10}$ It is worth to note, that if we compare eccentricities of low- and highmass planets considering all the planets with $M<4 M_{\text {Jup }}$ and $M>$ $4 M_{\text {Jup }}$ then the $P_{\mathrm{Ks}}$ decreases to $3 \times 10^{-5}$. 
single systems with masses between 1 and $5 M_{\text {Jup }}$ is generally damped due to planet-disk interaction, while for very massive planets with masses above $\sim 5 M_{\text {Jup }}$ the eccentricity can increase for low orbital inclinations relative to the disk. At the same time, $N$-body simulations of multiple giant planets performed by Raymond et al. (2010) and population synthesis models by Ida et al. (2013), and before that by Thommes et al. (2008), showed that the eccentricity increases with planetary mass. The latter authors explained this with a scenario in which multiple giant planets are mainly formed in relatively massive disks where dynamical instabilities, cohesive collisions, and orbit crossings are more common and can result in excitation of higher eccentricities.

Summarizing, Bitsch et al. (2013) predicted higher eccentricities for very high mass planets due to the interaction with disk (note that they performed their simulation for single-planet systems), and Ida et al. (2013) predicted correlation between $e$ and $M_{\mathrm{P}}$ for a wider range of planetary mass because of close scatterings and interaction of gas giants. We again applied a $\mathrm{K}-\mathrm{S}$ statistics to test whether the eccentricity distribution of planets with masses between 1 to $5 M_{\text {Jup }}$ and planets with $M_{\mathrm{P}}>$ $5 M_{\text {Jup }}$ are similar or not, but now we considered only planets in single systems. Our test predicts $P_{\mathrm{KS}} \approx 0.004$ that the two populations have the same underlying eccentricity distribution. We note that in the last test we considered only planets with $P>10$ days. If one consider planets with $P>50$ or $P>100$ days, or changes the $M_{\mathrm{P}}$ boundary to $4 M_{\mathrm{Jup}}$, the $P_{\mathrm{KS}}$ remains always smaller than 0.005 throughout.

Based on the observed trends, we can conclude that in general the eccentricities of high-mass planets, even in single-planet systems (although there is a possibility that in the systems there are more planets that are undetectable by current instrumentation and surveys or that some planets have been ejected), are higher than those for lower-mass planets, as predicted from the numerical simulation (Papaloizou et al. 2001; Bitsch et al. 2013).

Very recently, high-resolution near-infrared observation of HD100546 showed a variable ro-vibration of $\mathrm{CO}$ and $\mathrm{OH}$ emission lines (Liskowsky et al. 2012; Brittain et al. 2013), which the authors explained by postulating the presence of an eccentric massive planetary companion. This is an direct observational support of our results obtained statistically for a large sample.

\subsection{Orbital period}

The second correlation we observe is that very massive planets with eccentric orbits have longer periods than those with more circular orbits with $e<0.2$ (see Fig. 3). The K-S statistics predict $0.6 \%$ probability that the two families of planets have the same period distribution. This difference in periods is probably related to the interaction and migration processes in the disk that the planets suffered.

Cumming (2004) suggested that planets with long periods are on average easier to detect if their orbits are eccentric. However, our result cannot be explained with this observational selection effect because there is no similar correlation observed for lower-mass planets. The K-S statistics do not report a significant difference in period for planets with eccentric and circular orbits and with masses $1 M_{\text {Jup }}<M_{\mathrm{P}}<4 M_{\text {Jup }}$. The $P_{\mathrm{KS}}$ is 0.15 that low- and high-eccentricity planets with a mass in this interval have the same period distribution. This probability even increases from 0.15 to 0.76 when restricting the sample to periods longer than 10 to 100 days. Since the period distribution of planets with low $\left(1 M_{\text {Jup }}<M_{\mathrm{P}}<4 M_{\text {Jup }}\right)$ and high mass is simi$\operatorname{lar}\left(P_{\mathrm{KS}} \approx 0.65\right.$ for planets with $P>10$ days $)$, one could expect

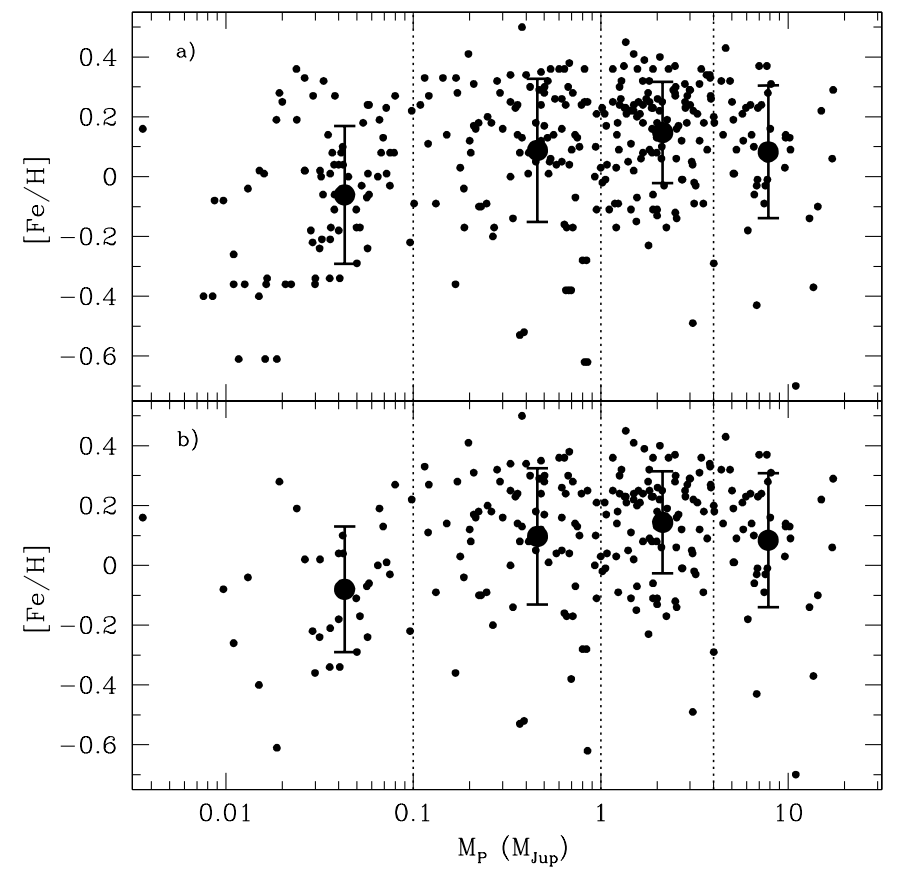

Fig. 4. Planetary mass against metallicty a) for all the planets in the system and b) for the most massive planets in each planetary systems. All the planets are detected with the radial velocity technique and planet hosts are FGK dwarf stars. The small circles correspond to individual planets and the large circles represent the average metallicty for the four mass bins $M_{\mathrm{P}}<0.1 M_{\text {Jup }} ; 0.1 M_{\text {Jup }} \leq M_{\mathrm{P}}<1 M_{\text {Jup }} ; 1 M_{\text {Jup }} \leq M_{\mathrm{P}}<$ $4 M_{\text {Jup }}$; and $M_{\mathrm{P}}>4 M_{\text {Jup }}$. The error bars indicate the standard deviation of the $[\mathrm{Fe} / \mathrm{H}]$ in each bin.

a more prominent correlation between eccentricity and period for lower-mass planets if there is a selection bias. This allows us to conclude that the correlation between $e$ and $\mathrm{P}$ for very high mass planets has a physical meaning, and does not come from an observational bias.

\subsection{Metallicity}

Interestingly, Ribas \& Miralda-Escudé (2007) found that the metallicity of planet host stars decreases with planet mass. In particular, they showed that the average metallicity of stars with planets of mass lower than $4 M_{\text {Jup }}$ is different from that of higher mass planet hosts at 3- $\sigma$ level, which is lower by about 0.15 dex. We again note that in their comparison they did not separate super-Earths and Neptunes, and in their sample almost all of the very low mass planets $\left(M_{\mathrm{P}}<0.3 M_{\mathrm{Jup}}\right)$ have $[\mathrm{Fe} / \mathrm{H}]>0$, while the majority of recently detected low-mass planets have subsolar or solar metallicities (e.g. Mayor et al. 2011; Buchhave et al. 2012).

Figure 4 shows the metallicities of FGK dwarf stars hosting RV-detected planets as a function of the planet mass. The metallicities were taken from the SWEET-Cat. On average, the metallicities of high-mass planets are similar. The average values and the standard error of the means are presented in the Table 1.

We tested the results obtained by Ribas \& Miralda-Escudé (2007) by applying a K-S test for the RV-detected sample of planets with masses between 1 and $4 M_{\text {Jup }}$ and with $M_{\mathrm{P}}>$ $4 M_{\text {Jup }}$. We obtained $P_{\mathrm{KS}} \approx 5.5 \%$ that the two subsamples have the same underlying metallicity distribution. If we consider only the planet with highest mass in the system, the probability increases to $15.3 \%$. We note that the metallicities of these 
Table 1. Average and standard errors of $[\mathrm{Fe} / \mathrm{H}]$ for planets with different masses: taking into account all the planets in the system (a) and only the most massive planet in the systems (b).

\begin{tabular}{lcc}
\hline \hline Planet mass & $<[\mathrm{Fe} / \mathrm{H}]>_{a}$ & $<[\mathrm{Fe} / \mathrm{H}]>_{b}$ \\
$M_{\text {Jup }}$ & dex & dex \\
\hline$M_{\mathrm{P}}<0.1$ & $-0.061 \pm 0.025$ & $-0.082 \pm 0.036$ \\
$0.1 \leq M_{\mathrm{P}}<1$ & $0.088 \pm 0.023$ & $0.096 \pm 0.025$ \\
$1 \leq M_{\mathrm{P}}<4$ & $0.149 \pm 0.016$ & $0.146 \pm 0.018$ \\
$M_{\mathrm{P}}>4$ & $0.083 \pm 0.032$ & $0.084 \pm 0.033$ \\
\hline
\end{tabular}

stars were taken from the literature, are not homogeneous, and include stars with different evolutionary stages. We again applied the same statistics, but taking only RV-detected planethost FGK dwarf stars for which SWEET-Cat provides homogeneous stellar parameters, including metallicity. In this case the difference in metallicity distribution between the two subsamples becomes even less significant with $P_{\mathrm{KS}} \approx 15 \%$ and $\approx 34.5 \%$ if one consider only the planet with the highest mass in each system. We therefore conclude that a simple separation in mass at $4 M_{\text {Jup }}$ does not reveal two different populations in metallicity, as hypothesized in Ribas \& Miralda-Escudé (2007). This result is expected from the core-accretion theory, since the metallicity mostly acts as a threshold for giant planet formation, but is not correlated with the mass of giant planets, except for planets with $M_{\mathrm{P}} \gtrsim 10-20 M_{\mathrm{Jup}}$, which are harder to form at clearly subsolar metallicities (Mordasini et al. 2012).

\section{Summary}

We analyzed the possible relations between orbital and physical properties of planets with different mass and physical properties of their host stars. The main findings and results are itemized below.

- We found that for a fixed maximum planetary mass between $10 M_{\oplus}$ and $4 M_{\text {Jup }}$, planets orbiting metal-poor stars are constrained to longer periods than those orbiting metalrich stars. Applied MC tests show that the obtained correlation is statistically significant. This result suggests that the mechanism responsible for the "separation" of planets in the $P-M_{\mathrm{P}}$ is operational for a wide range of planetary mass. The observed dependence can be explained by assuming that planets in a metal-poor disk form farther out from their central stars and/or do not migrate as far as planets in metalrich systems because they form later. Our result confirms the Beaugé \& Nesvorný (2013) findings and extends their conclusions to higher planetary masses.

- The Earth-like planets $\left(M_{\mathrm{P}}<10 M_{\oplus}\right)$ orbiting metal-rich stars have shorter periods than those orbiting metal-poor stars. If there is no detection bias, this could imply that the low-mass planets in metal-rich systems ordinarily migrate or that they always form close to their parent stars. The presence of Earth-like planets with short-periods orbiting metalpoor stars probably means that these planets can be formed close to their parent stars even at low metallicities because enough amount of protoplanetary mass is locally available.

- By applying a K-S test for a sample of FGK dwarf stars orbiting RV-detected planets we obtained $P_{\mathrm{KS}}$ up to $35 \%$ that the low- and high-mass planet hosts have the same underlying metallicity distribution. This result is expected in the core-accretion models (e.g. Mordasini et al. 2012), but it contradicts the results of Ribas \& Miralda-Escudé (2007), who found that the metallicity of planet-host stars decrease with planet mass.

- Analyzing the eccentricity distribution of high-mass planets, we found statistically significant evidence that planets with masses higher than $4 M_{\text {Jup }}$ have on average more highly eccentric orbits than the giant planets with masses between $1 M_{\text {Jup }}$ and $4 M_{\text {Jup }}$. These trends and dependencies agree with the core-accretion and formation in a disk models and allow us to conclude that planet-disk interaction is a very important and orbit-shaping mechanism when one moves towards higher planetary masses. In addition to these observational results, we found that less eccentric very high mass planets $\left(M_{\mathrm{P}}>4 M_{\mathrm{Jup}}\right)$ have shorter periods than those with similar mass that orbit their host stars with more highly eccentric orbits. This difference in periods is probably related to the interaction and migration processes in the disk that the planets underwent.

The dependencies and trends presented in this work can provide new constraints for the models and numerical simulations of planet formation and evolution. In particular the fact that almost all the giant planets orbiting metal-poor stars show long periods (i.e. longer than 100 days) shows that migration is less rapid than assumed in core-accretion planet formation models (e.g. Mordasini et al. 2012).

Acknowledgements. This work was supported by the European Research Council/European Community under the FP7 through Starting Grant agreement number 239953. V.Zh.A., S.G.S., E.D.M., and M.O. are supported by grants SFRH/BPD/70574/2010, SFRH/BPD/47611/2008, SFRH/BPD/76606/2011, and SFRH/BD/51981/2012 from the FCT (Portugal), respectively. A.C. is supported by grant PEst-C/CTM/LA0025/2011 from the FCT. C.M. acknowledges the support of the MPG through the Reimar-Lüst Fellowship. G.I. acknowledges financial support from the Spanish Ministry project MINECO AYA2011-29060. We gratefully acknowledge the anonymous referee for the constructive comments and suggestions.

\section{References}

Adibekyan, V. Z., Delgado Mena, E., Sousa, S. G., et al. 2012a, A\&A, 547, A36

Adibekyan, V. Z., Santos, N. C., Sousa, S. G., et al. 2012b, A\&A, 543, A89

Adibekyan, V. Z., Sousa, S. G., Santos, N. C., et al. 2012c, A\&A, 545, A32

Alibert, Y., Mordasini, C., Benz, W., \& Winisdoerffer, C. 2005, A\&A, 434, 343

Batygin, K. 2012, Nature, 491, 418

Beaugé, C., \& Nesvorný, D. 2012, ApJ, 751, 119

Beaugé, C., \& Nesvorný, D. 2013, ApJ, 763, 12

Bitsch, B., Crida, A., Libert, A.-S., \& Lega, E. 2013, A\&A, 555, A124

Boss, A. P. 1998, ApJ, 503, 923

Brittain, S. D., Najita, J. R., Carr, J. S., et al. 2013, ApJ, 767, 159

Brown, D. J. A., Collier Cameron, A., Díaz, R. F., et al. 2012, ApJ, 760, 139

Buchhave, L. A., Latham, D. W., Johansen, A., et al. 2012, Nature, 486, 375

Chiang, E., \& Laughlin, G. 2013, MNRAS, 431, 3444

Correia, A. C. M., Laskar, J., Farago, F., \& Boué, G. 2011, Celest. Mech. Dyn. Astron., 111, 105

Cumming, A. 2004, MNRAS, 354, 1165

D’Angelo, G., Lubow, S. H., \& Bate, M. R. 2006, ApJ, 652, 1698

Dawson, R. I., \& Murray-Clay, R. A. 2013, ApJ, 767, L24

Dawson, R. I., Murray-Clay, R. A., \& Johnson, J. A. 2012, ApJ, submitted [arXiv: 1211.0554]

Fabrycky, D., \& Tremaine, S. 2007, ApJ, 669, 1298

Figueira, P., Marmier, M., Boué, G., et al. 2012, A\&A, 541, A139

Fischer, D. A., \& Valenti, J. 2005, ApJ, 622, 1102

Ford, E. B., \& Rasio, F. A. 2006, ApJ, 638, L45

Fortier, A., Alibert, Y., Carron, F., Benz, W., \& Dittkrist, K.-M. 2013, A\&A, 549, A44

Gaudi, B. S. 2012, ARA\&A, 50, 411

Gonzalez, G. 2009, MNRAS, 399, L103

Halbwachs, J. L., Mayor, M., \& Udry, S. 2005, A\&A, 431, 1129

Hansen, B. M. S., \& Murray, N. 2012, ApJ, 751, 158 
A\&A 560, A51 (2013)

Hébrard, G., Bouchy, F., Pont, F., et al. 2008, A\&A, 488, 763

Ida, S., \& Lin, D. N. C. 2008, ApJ, 685, 584

Ida, S., Lin, D. N. C., \& Nagasawa, M. 2013, ApJ, 775, 42

Jorissen, A., Mayor, M., \& Udry, S. 2001, A\&A, 379, 992

Kley, W., \& Dirksen, G. 2006, A\&A, 447, 369

Lin, D. N. C., Bodenheimer, P., \& Richardson, D. C. 1996, Nature, 380, 606

Liskowsky, J. P., Brittain, S. D., Najita, J. R., et al. 2012, ApJ, 760, 153

Lodders, K. 2003, ApJ, 591, 1220

Lodders, K., Palme, H., \& Gail, H.-P. 2009, Landolt Börnstein - Group VI Astronomy and Astrophysics (Springer), 4.4

Lopez, S., \& Jenkins, J. S. 2012, ApJ, 756, 177

Lovis, C., Ségransan, D., Mayor, M., et al. 2011, A\&A, 528, A112

Mayor, M., \& Queloz, D. 1995, Nature, 378, 355

Mayor, M., Marmier, M., Lovis, C., et al. 2011, A\&A, submitted [arXiv: 1109.2497]

Mordasini, C., Alibert, Y., \& Benz, W. 2009a, A\&A, 501, 1139

Mordasini, C., Alibert, Y., Benz, W., \& Naef, D. 2009b, A\&A, 501, 1161

Mordasini, C., Klahr, H., Alibert, Y., Benz, W., \& Dittkrist, K.-M. 2010, A\&ARv, submitted [arXiv: 1012.5281]

Mordasini, C., Alibert, Y., Benz, W., Klahr, H., \& Henning, T. 2012, A\&A, 541, A97

Mortier, A., Santos, N. C., Sousa, S. G., et al. 2013, A\&A, 557, A70
Papaloizou, J. C. B., Nelson, R. P., \& Masset, F. 2001, A\&A, 366, 263

Pollack, J. B., Hubickyj, O., Bodenheimer, P., et al. 1996, Icarus, 124, 62

Rasio, F. A., \& Ford, E. B. 1996, Science, 274, 954

Raymond, S. N., Armitage, P. J., \& Gorelick, N. 2010, ApJ, 711, 772

Ribas, I., \& Miralda-Escudé, J. 2007, A\&A, 464, 779

Rogers, T. M., Lin, D. N. C., \& Lau, H. H. B. 2012, ApJ, 758, L6

Santos, N. C., Israelian, G., \& Mayor, M. 2001, A\&A, 373, 1019

Santos, N. C., Israelian, G., \& Mayor, M. 2004, A\&A, 415, 1153

Santos, N. C., Sousa, S. G., Mortier, A., et al. 2013, A\&A, 556, A150

Schneider, J., Dedieu, C., Le Sidaner, P., Savalle, R., \& Zolotukhin, I. 2011, A\&A, 532, A79

Socrates, A., Katz, B., Dong, S., \& Tremaine, S. 2012, ApJ, 750, 106

Sousa, S. G., Santos, N. C., Israelian, G., Mayor, M., \& Udry, S. 2011, A\&A, 533, A141

Sumi, T., Bennett, D. P., Bond, I. A., et al. 2010, ApJ, 710, 1641

Thommes, E. W., Matsumura, S., \& Rasio, F. A. 2008, Science, 321, 814

Triaud, A. H. M. J., Collier Cameron, A., Queloz, D., et al. 2010, A\&A, 524, A25

Udry, S., Mayor, M., Naef, D., et al. 2002, A\&A, 390, 267

Wolszczan, A., \& Frail, D. A. 1992, Nature, 355, 145

Wu, Y., \& Murray, N. 2003, ApJ, 589, 605

Xiang-Gruess, M., \& Papaloizou, J. C. B. 2013, MNRAS, 431, 1320 